\title{
A practical example demonstrating the utility of single-world intervention graphs
}

\author{
Alexander Breskin ${ }^{1}$, Stephen R. Cole ${ }^{1}$, and Michael G. Hudgens ${ }^{2}$ \\ ${ }^{1}$ Department of Epidemiology, University of North Carolina at Chapel Hill, Chapel Hill, NC, USA \\ ${ }^{2}$ Department of Biostatistics, University of North Carolina at Chapel Hill, Chapel Hill, NC, USA
}

\section{Keywords}

epidemiologic methods; selection bias; causal diagrams

\section{To the Editor}

Causal diagrams ${ }^{1,2}$ have become widespread in epidemiologic research. Recently developed single-world intervention graphs explicitly connect the potential outcomes framework of causal inference with causal diagrams. ${ }^{3}$ Here we provide a practical example demonstrating how single-world intervention graphs can supplement traditional causal diagrams.

A randomized controlled trial is conducted to evaluate whether a vaccine ( $A=1$ if vaccine, 0 if placebo) decreases the risk of disease ( $\mathrm{Y}=1$ if disease, 0 otherwise). Individuals are enrolled at baseline, randomized to vaccine or placebo, followed six months, and monitored for disease. The vaccine is more likely to result in injection site pain ( $\mathrm{W}=1$ if pain, 0 otherwise), and those with pain are more likely to drop out and have unobserved outcomes ( $\mathrm{S}=1$ if dropped out, 0 otherwise). Participants with poor (unmeasured) health ( $\mathrm{U}=1$ if poor health, 0 otherwise) are more likely to experience pain and get the disease. The scenario is summarized in Figure part a.

There is selection bias if we condition on not dropping out $(\mathrm{S}=0$ ) because the path $\mathrm{A} \rightarrow \mathrm{W}$ $\leftarrow \mathrm{U} \rightarrow \mathrm{Y}$ is opened. Stratifying on $\mathrm{W}$ does not block this path and may in fact induce more bias. Based on this causal diagram, it is not immediately clear how to identify the causal effect of the vaccine using the observed data (although see Bareinboim and Pearl $2012^{4}$, Bareinboim, Tian, and Pearl $2014^{5}$, or Bareinboim and Tian $2015^{6}$ ).

The single-world intervention graph in Figure part b, however, clearly displays the independencies necessary to identify the effect of the vaccine from the observed data as follows (here, a variable $\mathrm{X}(\mathrm{a})$ represents the value of $\mathrm{X}$ had the individual received vaccine level a):

Correspondence to: Alexander Breskin, Department of Epidemiology, University of North Carolina at Chapel Hill, CB 7435 McGavran-Greenberg Hall, Chapel Hill, NC 27599, abreskin@unc.edu, (917) 5939004.

Conflicts of interest: The authors declare no conflicts of interest

Availability of Code: The computing code is available in the eAppendix for this letter. 


$$
\begin{aligned}
& E(Y(a))=\sum_{w} E(Y(a) \mid W(a)=w) P(W(a)=w)=\sum_{w} E(Y(a) \mid W(a)=w, S(a)=0) P(W(a)=w) \\
& =\sum_{w} E(Y(a) \mid W(a)=w, S(a)=0) P(W(a)=w \mid A=a)=\sum_{w} E(Y(a) \mid W(a)=w, S(a)=0, A=a) P(W(a)=w \mid A=a \\
& )=\sum_{w} E(Y \mid W=w, S=0, A=a) P(W=w \mid A=a)
\end{aligned}
$$

The first equality holds by the law of total probability, the second by d-separation of S(a) and $\mathrm{Y}(\mathrm{a})$ given $\mathrm{W}(\mathrm{a})$, the third by d-separation of $\mathrm{W}(\mathrm{a})$ and $\mathrm{A}$, the fourth by d-separation of Y(a) and A given W(a) and S(a), and the last by causal consistency. All components of the final line of the equation, which is Robins' $g$-formula ${ }^{7}$, can be estimated from observed data. The key insight provided by the single-world intervention graph is that $S(a)$ is independent of $\mathrm{Y}(\mathrm{a})$ given $\mathrm{W}(\mathrm{a})$, but conditioning on W(a) does not open any paths between $\mathrm{A}$ and $\mathrm{Y}(\mathrm{a})$.

We conducted a simulation of 1,000,000 individuals for illustration (SAS code is available in the eAppendix). Individuals were randomly assigned vaccine with probability 0.5 and had probability 0.3 of being in poor health. The probability of injection site pain for healthy individuals was 0.2 if assigned placebo and 0.6 if assigned vaccine. Poor health increased the probability of pain by 0.3 . The probability of dropping out was 0.1 for those without pain and 0.9 for those with pain. Finally, the probability of disease was 0.3 for healthy individuals assigned placebo, and it was increased by 0.5 by poor health and decreased by 0.2 by the vaccine.

The true effect of the vaccine on the disease was a 0.20 decrease in risk. The complete case analysis gave a 0.24 decrease in risk. Stratifying on injection site pain worsened the bias, giving a 0.26 decrease in risk. Finally, the g-formula with empirically estimated expectations and probabilities yielded the true decrease of 0.20 .

An anonymous reviewer noted that the derivation above also holds with certain additional edges in the causal diagram, such as $\mathrm{W} \rightarrow \mathrm{Y}$ or $\mathrm{A} \rightarrow \mathrm{S}$. These would lead to, respectively, edges $\mathrm{W}(\mathrm{A}) \rightarrow \mathrm{Y}(\mathrm{a})$ or a $\rightarrow \mathrm{S}(\mathrm{a})$ in the single-world intervention graph. In the latter case, $\mathrm{S}(\mathrm{a})$ is d-seperated from $\mathrm{Y}(\mathrm{a})$ given $\mathrm{W}(\mathrm{a})$ and a, thus $\mathrm{S}(\mathrm{a})$ would remain independent of $\mathrm{Y}(\mathrm{a})$ conditional on W(a) (Richardson and Robins, Theorem $12^{3}$ ). The reviewer also noted that the derivation fails with unmeasured confounding between $\mathrm{A}$ and $\mathrm{W}$ or between $\mathrm{S}$ and $\mathrm{Y}$.

\section{Supplementary Material}

Refer to Web version on PubMed Central for supplementary material.

\section{Acknowledgments}

The authors would like to thank the anonymous reviewer of this letter for their helpful comments.

Financial Support: This work was supported by the National Institutes of Health grants R01AI100654 (SRC) and R01AI085073 (MGH)

\section{References}

1. Pearl J. Causal diagrams for empirical research. Biometrika. 1995; 82(4):669-688. 
2. Greenland S, Pearl J, Robins JM. Causal diagrams for epidemiologic research. Epidemiology. 1999; 10(1):37-48. [PubMed: 9888278]

3. Richardson TS, Robins JM. Single world intervention graphs (SWIGs): A unification of the counterfactual and graphical approaches to causality. Cent Stat Soc Sci Univ Washingt Ser Work Pap. 2013; 128(30):2013.

4. Bareinboim E, Pearl J. Controlling selection bias in causal inference. Artificial Intelligence and Statistics. 2012:100-108.

5. Bareinboim E, Tian J, Pearl J. Recovering from Selection Bias in Causal and Statistical Inference. AAAI. 2014:2410-2416.

6. Bareinboim E, Tian J. Recovering Causal Effects from Selection Bias. AAAI. 2015:3475-3481.

7. Robins J. A new approach to causal inference in mortality studies with a sustained exposure period —application to control of the healthy worker survivor effect. Math Model. 1986; 7(9-12):13931512 . 
A
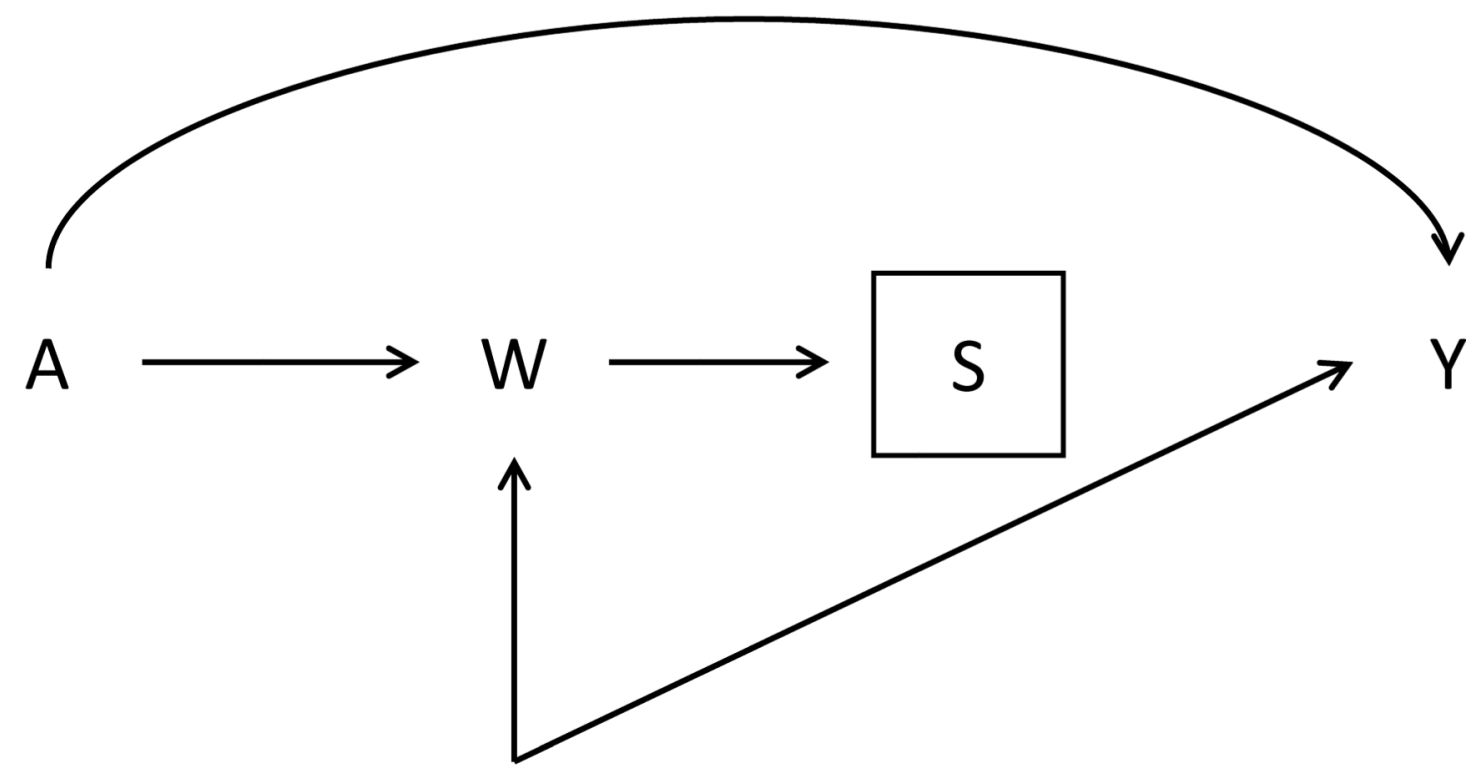

B

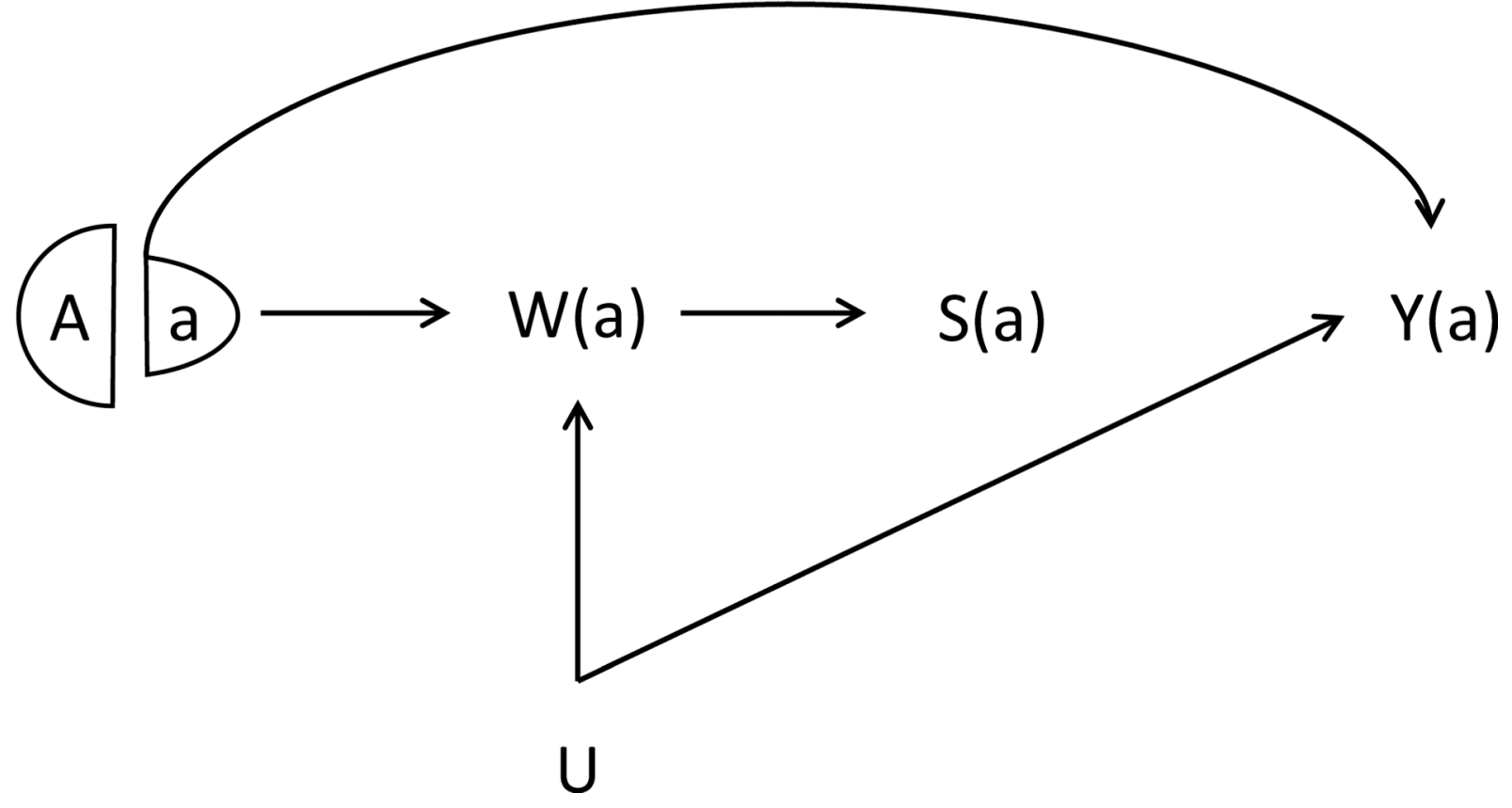

Figure.

The causal diagram (a) corresponding to the vaccine trial. The single-world intervention template (b), the template used to construct single-world intervention graphs, corresponding 
to the vaccine trial is constructed by splitting the treatment node of the causal diagram, and replacing all descendants of the assigned treatment with their potential outcomes. 